\title{
Epigenetic Downregulation of Atrial Natriuretic Peptide but not Vasopressin mRNA Expression in Females with Eating Disorders is Related to Impulsivity
}

\author{
Helge Frieling*, ${ }^{*, 4}$, Stefan Bleich 1,4, Jeannette Otten', Konstanze D Römer', Johannes Kornhuber', \\ Martina de Zwaan ${ }^{2}$, Georg E Jacoby ${ }^{3}$, Julia Wilhelm' and Thomas Hillemacher' \\ 'Department of Psychiatry and Psychotherapy, Friedrich-Alexander-University Erlangen-Nuremberg, Erlangen, Germany; ${ }^{2}$ Department of \\ Psychosomatic Medicine and Psychotherapy, Friedrich-Alexander-University Erlangen-Nuremberg, Erlangen, Germany; ${ }^{3}$ Klinik am Korso, Hospital \\ for Eating Disorders, Bad Oeynhausen, Germany
}

\begin{abstract}
Disturbances of volume-regulating mechanisms have already been implicated in the pathophysiology of eating disorders like anorexia or bulimia nervosa with the peptide hormones vasopressin and atrial natriuretic peptide (ANP) being of special interest. Aim of the present study was to investigate, whether the expression of the corresponding genes was altered and if so, if these changes could be explained by epigenetic mechanisms such as DNA methylation. We analyzed blood samples of 46 women suffering from anorexia $(n=22)$ or bulimia nervosa $(n=24)$ as well as of 30 healthy controls. Peripheral mRNA expression and DNA methylation of the vasopressin and the ANP precursor genes were assessed using real-time PCR. We found significantly lower levels of ANP mRNA in patients with eating disorders. This downregulation was accompanied by a hypermethylation of the ANP gene promoter in the bulimic subgroup. We did not find differences regarding expression or methylation of the vasopressin gene. ANP mRNA expression was inversely associated with impaired impulse regulation. We conclude that epigenetic mechanisms may contribute to the known alterations of ANP homeostasis in women with eating disorders.

Neuropsychopharmacology (2008) 33, 2605-2609; doi:I0.1038/sj.npp. I 30 I662; published online 2 January 2008
\end{abstract}

Keywords: eating disorders; vasopressin; atrial natriuretic peptide; mRNA; DNA methylation; EDI-2

\section{INTRODUCTION}

The recent years have witnessed a renewed interest in alterations of volume- and appetite-regulating hormones in several psychiatric disorders, like depression, anxiety (Strohle and Holsboer, 2003), and alcohol dependence (Hillemacher et al, 2006a,b). Even though the first reports date back into the sixties and seventies of the last century (Aperia et al, 1978; Russell and Bruce, 1966), the role of volume-regulating hormones and peptides in the etiology of eating disorders has found increasing attention during the last decade (Kaye, 1996).

Among these hormones, atrial natriuretic peptide (ANP) and vasopressin have been most extensively studied, as both are known to exert effects in the CNS. Vasopressin enhances memory function and can have anxiogenic effects. It is

*Correspondence: Dr $\mathrm{H}$ Frieling, Department of Psychiatry and Psychotherapy, Friedrich-Alexander-University Erlangen-Nuremberg, Schwabachanlage 6, 91054 Erlangen, Germany, Tel: + 49 9131 853 4262, Fax: + 49 9131 853 4105, E-mail: helge.frieling@uk-erlangen.de ${ }^{4}$ These authors contributed equally to this work

Received 17 August 2007; revised 19 November 2007; accepted 25 November 2007 implicated in the regulation of feeding and in obsessional behavior and also depression (reviewed in Kaye, 1996). Elevated levels of vasopressin have been found in the CSF of patients with acute illness or after recovery from anorexia nervosa (AN) and bulimia nervosa (BN) (Frank et al, 2000; Gold et al, 1983).

ANP has potent anxiolytic effects and is a strong inhibitor of the effects of corticotropin releasing hormone, corticotropin and cortisol (Strohle et al, 2001, 2006; Wiedemann et al, 2001). Dysregulation of ANP plasma levels have been implicated in the pathophysiology of depression and protracted alcohol withdrawal (Kiefer et al, 2002; Strohle and Holsboer, 2003). Elevated plasma levels of ANP have been reported in women suffering from AN (Ohashi et al, 1988) as well as an impaired response of ANP to an acute water load (Baranowska et al, 1995). To our knowledge, no results have been published regarding patients suffering from BN.

As both hormones are peptides, the long-term regulation of their expression may underlie epigenetic mechanism, such as DNA methylation and/or histone acetylation. We have recently reported disturbances in DNA methylation in women with eating disorders (Frieling et al, 2007), that may 
also underlie the known alterations of vasopressin and ANP homeostasis in these disorders. The present exploratory study was conducted to evaluate whether mRNA expression and/or DNA promoter methylation of the ANP precursor gene $(N P P A)$ or the vasopressin precursor gene $(A V P)$ are present in women with eating disorders. As some neurobiological differences exist between restrictive type and bulimic type anorectic patients on the one hand and on the other hand bulimic type anorectic patients resemble subjects with BN more closely in other aspects, it seems reasonable to evaluate subgroups of the eating disorders (Bailer et al, 2007; Mercader et al, 2007). Unfortunately, the sample size of our study prevents subgroup analyses. Therefore, all patients are to be analyzed according to their diagnosis (AN/BN) or according to the presence of binging or purging behavior.

\section{MATERIALS AND METHODS}

\section{Patients and Design}

This study was part of an ongoing observational study on homocysteine and DNA methylation in eating disorders (Frieling et al, 2005, 2007). It was approved by the local ethics committee (University of Münster, Germany). All patients met DSM-IV criteria for AN or BN. Diagnoses were established using a structured interview. Comorbid psychiatric disorders were assessed using the German version of the Structured Clinical Interview for DSM-IV diagnoses. Written informed consent was obtained from all patients after the procedures had been fully explained. All patients were inpatients in a psychosomatic hospital (Korso Hospital, Bad Oeynhausen, Germany). Fasting blood samples were drawn on the day after admission to the hospital for routine and genetic analyses. To assess possible fluid alterations, serum electrolytes, hematocrit and creatinine were assessed. In the present analysis we included 22 women with AN (age: 22 years (18-51); BMI: 15.9(2.0)), 24 women with BN (age: 23 (18-42); BMI: 22.6 (2.6)), and compared the measurements with 30 age-matched healthy women as controls (age: 21 (19-43); BMI: 20.3 (4.1)). Two experienced psychiatrists (SB and TH) examined all control subjects prior to enrolment to ensure that they did not suffer from any psychiatric disorder or behavioral disturbance. Healthy women were recruited during lectures held by us.

\section{Psychometric Assessment}

Eating disorder symptomatology was assessed with the German version of the Eating Disorder Inventory 2 (EDI-2), a self-rating instrument consisting of 91 items and 11 subscales. It was designed for the assessment of attitudinal and behavioral dimensions relevant to $\mathrm{AN}$ and $\mathrm{BN}$ (Thiel et al, 1997). The EDI-2 consists of the eight original subscales: drive for thinness, bulimia (B), body dissatisfaction, ineffectiveness, perfectionism, interpersonal distrust, interoceptive awareness and maturity fears, and three new subscales: asceticism, impulse regulation (IR), and social insecurity. EDI-2 sum scores were used to estimate the severity of the disorder.

\section{Laboratory Analysis}

Analysis of DNA Methylation. Total DNA was extracted from frozen EDTA blood using QIAmp DNA Blood Mini Kit (Qiagen, Hilden, Germany). Promoter-specific DNA methylation status was measured using a methylation-specific endonuclease digestion followed by real-time PCR as previously described (Bleich et al, 2006). Primer sequences and annealing temperatures are provided in Table 1. Oligos were obtained from Operon (Operon $\mathrm{GmbH}$, Cologne, Germany).

Analysis of mRNA Expression. Total RNA was extracted from whole frozen EDTA blood using a standard phenolchloroform extraction in Qiazol (Qiagen), followed by column purification with Rneasy Mini Kit (Qiagen), including DNase digestion. After reverse transcription using the iScript cDNA Synthesis System (Bio-Rad, Hercules, CA), quantitative PCR was performed using iQ SYBR Green Super Mix buffer (Bio-Rad). GAPDH was used as an internal standard, and $\Delta C_{\mathrm{T}}$ values were calculated from differences between the analyzed genes and GAPDH.

\section{Statistical Analysis}

All parameters were normally distributed according to the Kolmogorov-Smirnov test, but age had a kurtosis, which was discovered in the graphic analysis. For the regression analyses, we therefore used the ln of age. Between-group comparisons were done using either Student's $t$-test (for two groups) or one-way analysis of variance (ANOVA) with Bonferroni's post hoc test for three or more groups. Measurements of expression and DNA methylation failed

Table I Oligonucleotides Used as Primers

\begin{tabular}{|c|c|c|c|c|}
\hline Name & Forward & Backward & $\begin{array}{l}\text { Annealing } \\
\left.\text { temp ( }{ }^{\circ} \mathrm{C}\right)\end{array}$ & $\begin{array}{l}\text { Product } \\
\text { length (bp) }\end{array}$ \\
\hline Vasopressin_exp & TGCATACGGGGTCCACCTGT & TAGTTCTCCTCCTGGCAGC & 57 & 271 \\
\hline GAPDH & CTGGAACGGTGAAGGTGACA & AAGGGACTTCCTGTAACAATGCA & 57 & 139 \\
\hline Vasopressin_meth & CTTTAGACCTGCCACCTTGG & CCAGGGCAGGTACATCTCAG & 60 & 150 \\
\hline
\end{tabular}

Abbreviations: ANP, atrial natriuretic peptide; GAPDH, glyceraldehyde-3-phosphate dehydrogenase. 
in some cases despite repeated trials. Therefore reported degrees of freedom may differ for different results. Categorical data was assessed using Pearson's $\chi^{2}$ test. To further analyze the results we used linear regression analyses and a multivariate general linear model incorporating the EDI-2 subscales as dependent variables and the $\Delta C_{\mathrm{T}}$ values of vasopressin and ANP as independent variables. The results are presented as means (SD), with age as only exception being reported as median (range). $P$-values of less than 0.05 (two tailed) were considered to indicate statistical significance. Data were analyzed employing SPSS for Windows 14.0 (SPSS Inc., Chicago, IL) and GraphPad Prism 4 (GraphPad Software Inc., San Diego, CA).

\section{RESULTS}

\section{Analysis of Gene-Specific DNA Methylation and Expression}

Patients suffering from both, $\mathrm{AN}$ and $\mathrm{BN}$, had significantly lower levels of ANP mRNA (AN: $\Delta C_{\mathrm{T}}-3.26 \pm 1.4 ; \mathrm{BN}: \Delta C_{\mathrm{T}}$ $-3.30 \pm 1.7$; ANOVA: $\mathrm{F}=4.26$; d.f. $=65 ; P=0.018)$ than controls $\left(\Delta C_{\mathrm{T}}-1.64 \pm 3.1\right)$ (Figure 1a). Expression of
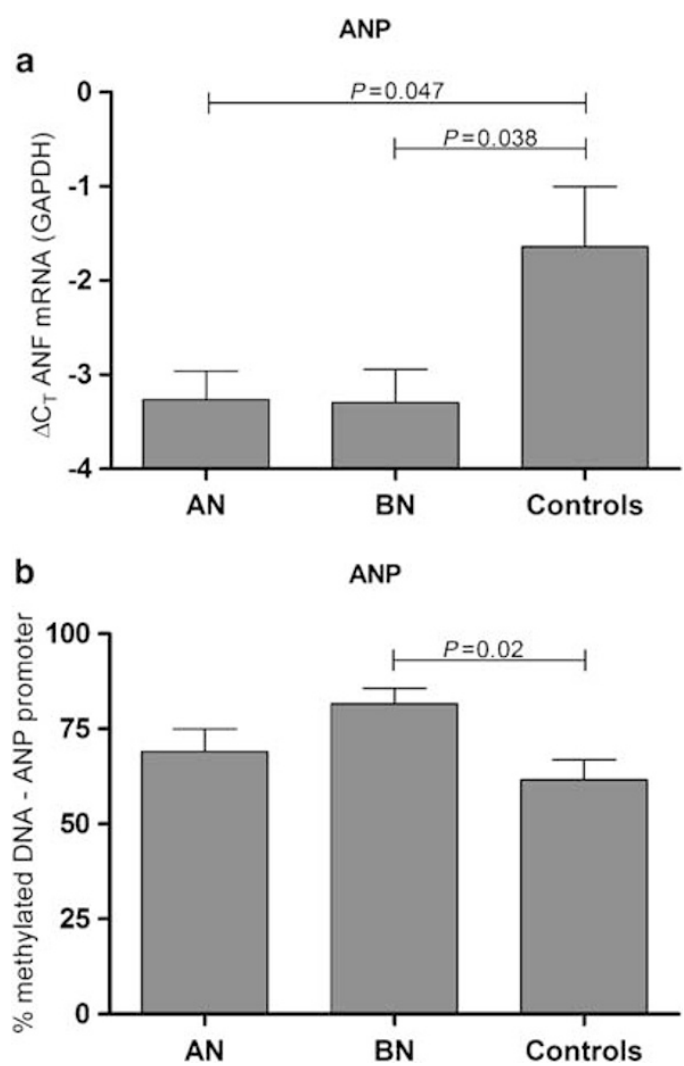

Figure I mRNA expression and DNA methylation of the atrial natriuretic precursor peptide gene. Women suffering from anorexia (AN) or bulimia nervosa (BN) have a significantly lower mRNA expression in the blood than healthy women (a). This downregulation is partially mediated by epigenetic DNA hypermethylation of the corresponding gene's promoter structure (b). P-values indicated in the figure are derived from Bonferroni's post hoc test after one-way analysis of variance (ANOVA). Further statistical details are summarized in the 'Results' and 'Materials and Methods' sections. vasopressin did not differ between groups (AN: $\Delta C_{\mathrm{T}} \quad-8.72 \pm 3.4 ; \quad \mathrm{BN}: \quad \Delta C_{\mathrm{T}} \quad-6.97 \pm 2.7 ; \quad$ controls: $\Delta C_{\mathrm{T}}-8.26 \pm 1.9 ;$ ANOVA: $\mathrm{F}=2.28$; d.f. $=60 ; P=0.111$ ).

As shown in Figure 1b, we found a significantly higher DNA methylation of the ANP gene promoter in patients with BN (81.53 $\pm 20.1 \%$; ANOVA: $\mathrm{F}=3.96$; d.f. $=69$; $P=0.024)$ compared to controls $(61.54 \pm 27.2 \%)$. Patients with AN $(68.93 \pm 27.9 \%)$ did not differ from patients with $\mathrm{BN}$ and controls ( $P$-values of the post hoc tests are provided in the figure). Comparing all patients (AN and $\mathrm{BN}$ ) that exhibit purging behavior against those with restrictive or nonpurging types and controls, also a significant difference was found in the DNA methylation (purging: $78.53 \pm 20.8 \%$; nonpurging: $64.64 \pm 34.4 \%$; ANOVA: $\mathrm{F}=3.68$; d.f. $=69$; $P=0.030$; Bonferroni: purging $v s$ nonpurging: $P=0.39$; purging $v s$ control: $P=0.034$; nonpurging $v s$ control: $P=0.99$ ). An analysis based on the presence of binging behavior failed to produce significant differences between the groups (data not shown). No significant differences were found in the amount of DNA methylation in the vasopressin promoter (AN: $82.85 \pm 15.3 \%$; BN: $85.10 \pm 15.4 \%$; controls: $82.31 \pm 18.1 \%$; ANOVA: $\mathrm{F}=0.19$; d.f. $=66 ; P=0.83)$.

\section{Association with Clinical Variables}

Neither the expression of ANP nor vasopressin was correlated with BMI, age, or duration of illness (data not shown). BMI and age were correlated in the BN and control group, age and duration of illness were correlated in the AN and BN group (data not shown). Partial correlation controlling for these correlation also produced no meaningful results for both, ANP or vasopressin expression. Measurements of serum electrolytes, hematocrit and creatinine did not show main alterations in the patients. We neither found an association between these variables and the expression nor the methylation of ANP and vasopressin.

Analyzing the patients group only, we found an association between the genes' expression and the EDI-2 sum score. Entering both variables into the regression model, only the expression of ANP was inversely associated with the EDI-2 score $(B=-9.68 ; P=0.038$; Figure $2 \mathrm{a})$. In the multivariate regression model that incorporated all EDI-2 subscales as dependent and the genes' expression as independent variables, a negative linear relationship was found for ANP and disturbed IR $(B=-1.99 ; P=0.001$; Figure 2b).

\section{DISCUSSION}

To our knowledge, this is the first report of altered gene expression of the ANP gene in eating disorders. We found elevated DNA methylation of the ANP gene promoter to underlie the decreased expression of its mRNA. We found a significant hypermethylation only in the BN patients, however this may be explained by the small sample size. Our findings suggest that the observed differences in methylation of ANP are connected to purging behavior. Therefore it may be that the result for the AN group would be different if looking at restrictive type anorexia only. This is in line with several other findings, reporting disturbed 

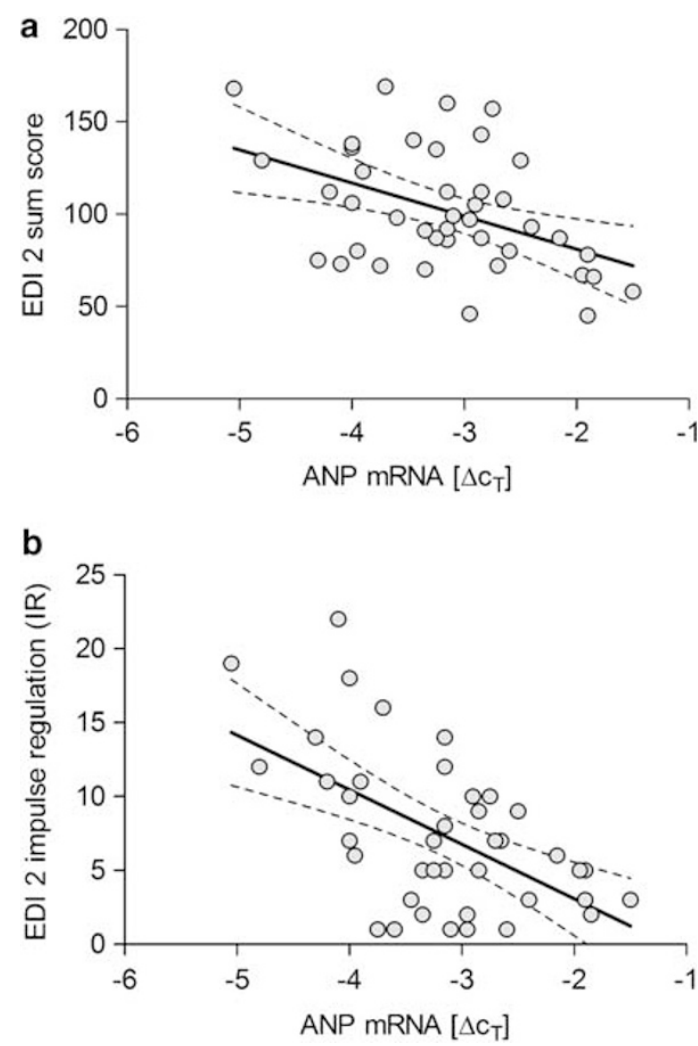

Figure 2 Scatter plot of the relation between atrial natriuretic precursor peptide gene's expression and scores of the eating disorder inventory 2 (EDI-2). The figures show the relation between the mRNA expression of atrial natriuretic peptide (ANP) and the sum score (a) or the impulse regulation (IR) disturbance score (b) of the EDI-2. The straight line represents the regression curve of a simple linear regression with it's $95 \%$ confidence interval (dashed lines).

levels of neuropeptides only in the bulimic subtype of AN (Fujimoto et al, 1997). Still, further studies are needed to replicate our findings, especially concerning the subtypes of AN and BN, which the sample size of our study did not permit. It seems likely that changes as those observed in our study may not be present in the restrictive type of AN.

Our finding of a downregulation of the ANP mRNA expression is in apparent contradiction to findings of elevated plasma levels of the peptide itself, at least in patients with AN (Ohashi et al, 1988). Still, some explanations for this finding may be found. It seems reasonable that elevated levels of ANP may induce their own downregulation. ANP exerts its effects at least partially via the activation of the nitric oxide (NO) - cyclic guanosine monophosphate pathway, which has been implicated in the control of DNA methylation: NO directly increases the activity of several DNA methyltransferases leading to hypermethylation of certain genes (Hmadcha et al, 1999). Cyclic nucleotides have also been shown to directly interfere with DNA methylation (Fedorov and Ermil'chenko, 1980). But even if elevated ANP leads to a downregulation of its own gene expression, the elevated levels themselves need to be explained. A possible solution may be found in the decreased activity of several peptidase enzymes in patients with eating disorders, among them dipeptidylpeptidase IV, an enzyme that also degrades ANP (Brandt et al, 2006; West et al, 2000). As dipeptidylpeptidase itself is also regulated by DNA methylation (Ballestar et al, 2003), it is tempting to speculate that a vicious circle of hypermethylation leading to decreased peptidase activity further pronouncing hypermethylation may be built. This speculation, however, needs to be verified in preclinical studies, which in fact are underway.

The clinical relevance of our finding is underscored by the apparent association of ANP mRNA levels and disturbed IR showing that a more 'normal' ANP expression is associated with less impulsivity. More dysregulated volume hormones seems to be associated with more severe forms of the disorder, as indicated by the EDI-2 sum scores. However, as we only report a cross-sectional analysis, caution is warranted. It is not clear, if ANP is causally involved in the disturbed IR, it could also just serve as a surrogate for other downregulated genes directly influencing impulse control.

Our negative finding regarding vasopressin despite studies reporting elevated levels of the peptide in the peripheral blood of patients with eating disorders (Kaye, 1996) may be explainable by the fact that unlike ANP, vasopressin is exclusively secreted by the posterior pituitary. Therefore, measurements of its mRNA in peripheral blood may only be of limited relevance. However little is known about the contributions of epigenetic mechanisms on the homeostasis of vasopressin, an issue further studies should address.

While designing the study we also expected to find fluid and electrolyte alterations in patients suffering from eating disorders, as this would be the best explanation for the disturbances in hormones regulating the volume homeostasis. Contrary to this assumption, no major alterations of serum electrolytes, hematocrit, or creatinine were present in our sample and no association with the volume-regulating hormones was found. This casts some doubts on the hypothesis that these hormonal systems are exclusively involved in the volume regulation. It seems more likely that other mechanisms associated with the disorders are influencing the expression at least of ANP.

In conclusion, we report further evidence for the hypothesis that disturbances in some hormones of the volume-regulating systems may contribute to eating disorder pathology. Our study may advance our understanding of epigenetic mechanisms involved in the pathophysiology of these disorders. Additional studies, preclinical as well as clinical, are needed to further investigate the interaction of hormonal peptides, DNA methylation, gene expression, and clinical signs of the disorder.

\section{ACKNOWLEDGEMENTS}

We thank Franziska Mittelbach and Sarah Scholz for excellent technical support for the laboratory analyses. HF and SB were supported by a grant by the Interdisciplinary Centre for Clinical Research (IZKF, University of ErlangenNuremberg).

\section{DISCLOSURE/CONFLICTS OF INTEREST}

The authors have nothing to disclose. 


\section{REFERENCES}

Aperia A, Broberger O, Fohlin L (1978). Renal function in anorexia nervosa. Acta Paediatr Scand 67: 219-224.

Bailer UF, Frank GK, Henry SE, Price JC, Meltzer CC, Becker C et al (2007). Serotonin transporter binding after recovery from eating disorders. Psychopharmacology (Berl) 195: 315-324.

Ballestar E, Paz MF, Valle L, Wei S, Fraga MF, Espada J et al (2003). Methyl-CpG binding proteins identify novel sites of epigenetic inactivation in human cancer. EMBO J 22: 6335-6345.

Baranowska B, Wasilewska-Dziubinska E, Radzikowska M, Plonowski A, Roguski K (1995). Impaired response of atrial natriuretic peptide to acute water load in obesity and in anorexia nervosa. Eur J Endocrinol 132: 147-151.

Bleich S, Lenz B, Ziegenbein M, Beutler S, Frieling H, Kornhuber J et al (2006). Epigenetic DNA hypermethylation of the HERP gene promoter induces down-regulation of its mRNA expression in patients with alcohol dependence. Alcohol Clin Exp Res 30: 587-591.

Brandt I, Lambeir AM, Ketelslegers JM, Vanderheyden M, Scharpe S, De Meester I (2006). Dipeptidyl-peptidase IV converts intact B-type natriuretic peptide into its des-SerPro form. Clin Chem 52: 82-87.

Fedorov NA, Ermil'chenko GV (1980). Effect of cyclic nucleotides and hormones of homologous methylation in nuclear homogenates of rat bone marrow. Biokhimiia 45: 1048-1051.

Frank GK, Kaye WH, Altemus M, Greeno CG (2000). CSF oxytocin and vasopressin levels after recovery from bulimia nervosa and anorexia nervosa, bulimic subtype. Biol Psychiatry 48: 315-318.

Frieling H, Gozner A, Römer KD, Lenz B, Bönsch D, Wilhelm J et al (2007). Global DNA hypomethylation and DNA hypermethylation of the alpha synuclein promoter in females with anorexia nervosa. Mol Psychiatry 12: 229-230.

Frieling H, Römer K, Röschke B, Bönsch D, Wilhelm J, Fiszer R et al (2005). Homocysteine plasma levels are elevated in females with anorexia nervosa. J Neural Transm 112: 979-985.

Fujimoto S, Inui A, Kiyota N, Seki W, Koide K, Takamiya S et al (1997). Increased cholecystokinin and pancreatic polypeptide responses to a fat-rich meal in patients with restrictive but not bulimic anorexia nervosa. Biol Psychiatry 41: 1068-1070.

Gold PW, Kaye W, Robertson GL, Ebert M (1983). Abnormalities in plasma and cerebrospinal-fluid arginine vasopressin in patients with anorexia nervosa. New Engl J Med 308: 1117-1123.
Hillemacher T, Bayerlein K, Wilhelm J, Poleo D, Frieling $\mathrm{H}$, Ziegenbein $\mathrm{M}$ et al (2006a). Volume intake and craving in alcohol withdrawal. Alcohol Alcohol 41: 61-65.

Hillemacher T, Bleich S, Frieling H, Schanze A, Wilhelm J, Sperling W et al (2006b). Evidence of an association of leptin serum levels and craving in alcohol dependence. Psychoneuroendocrinology 32: 87-90.

Hmadcha A, Bedoya FJ, Sobrino F, Pintado E (1999). Methylationdependent gene silencing induced by interleukin $1 \beta$ via nitric oxide production. J Exp Med 190: 1595-1604.

Kaye WH (1996). Neuropeptide abnormalities in anorexia nervosa. Psychiatry Res 62: 65-74.

Kiefer F, Andersohn F, Jahn H, Wolf K, Raedler TJ, Wiedemann K (2002). Involvement of plasma atrial natriuretic peptide in protracted alcohol withdrawal. Acta Psychiatr Scand 105: 65-70.

Mercader JM, Ribases M, Gratacos M, Gonzalez JR, Bayes M, de Cid $\mathrm{R}$ et al (2007). Altered brain-derived neurotrophic factor blood levels and gene variability are associated with anorexia and bulimia. Genes Brain Behav 6: 706-716.

Ohashi M, Fujio N, Nawata H, Kato K, Matsubayashi S, Tamai H et al (1988). Human atrial natriuretic polypeptide in plasma of patients with anorexia nervosa. Horm Metab Res 20: 705-708.

Russell GFM, Bruce JT (1966). Impaired water diuresis in patients with anorexia nervosa. Am J Med 40: 38-48.

Strohle A, Feller C, Strasburger CJ, Heinz A, Dimeo F (2006). Anxiety modulation by the heart? Aerobic exercise and atrial natriuretic peptide. Psychoneuroendocrinology 31: 1127-1130.

Strohle A, Holsboer F (2003). Stress responsive neurohormones in depression and anxiety. Pharmacopsychiatry 36(Suppl 3): S207-S214.

Strohle A, Kellner M, Holsboer F, Wiedemann K (2001). Anxiolytic activity of atrial natriuretic peptide in patients with panic disorder. Am J Psychiatry 158: 1514-1516.

Thiel A, Jacobi C, Horstmann S, Paul T, Nutzinger DO, Schussler G (1997). A German version of the eating disorder inventory EDI-2. Psychother Psychosom Med Psychol 47: 365-376.

West D, Monteleone P, Lieto AD, Meester ID, Durinx C, Scharpe S et al (2000). Lowered serum dipeptidyl peptidase IV activity in patients with anorexia and bulimia nervosa. Eur Arch Psychiatry Clin Neurosci 250: 86-92.

Wiedemann K, Jahn H, Yassouridis A, Kellner M (2001). Anxiolyticlike effects of atrial natriuretic peptide on cholecystokinin tetrapeptide-induced panic attacks: preliminary findings. Arch Gen Psychiatry 58: 371-377. 